TRANSACTIONS OF THE

AMERICAN MATHEMATICAL SOCIETY

Volume 179, May 1973

\title{
THE MEASURE ALGEBRA OF A LOCALLY COMPACT HYPERGROUP
}

\author{
BY \\ CHARLES F. DUNKL(1)
}

\begin{abstract}
A hypergroup is a locally compact space on which the space of finite regular Borel measures has a convolution structure preserving the probability measures. This paper deals only with commutative hypergroups. $\$ 1$ contains definitions, a discussion of invariant measures, and a characterization of idempotent probability measures. $\$ 2$ deals with the characters of a hypergroup. $\$ 3$ is about hypergroups, which have generalized translation operators (in the sense of Levitan), and subhypergroups of such. In this case the set of characters provides much information. Finally $\$ 4$ discusses examples, such as the space of conjugacy classes of a compact group, certain compact homogeneous spaces, ultraspherical series, and finite hypergroups.
\end{abstract}

A hypergroup is a locally compact space on which the space of finite regular Borel measures has a convolution structure preserving the probability measures. Such a structure can arise in several ways in harmonic analysis. Two major examples are furnished by the space of conjugacy classes of a compact nonabelian group, and by the two-sided cosets of certain nonnormal closed subgroups of a compact group. Another example is given by series of Jacobi polynomials.

The class of hypergroups includes the class of locally compact topological semigroups. In this paper we will show that many well-known group theorems extend to the commutative hypergroup case. In $\$ 1$ we discuss some basic structure and determine the idempotent probability measures. In $\$ 2$ we present some elementary theory of characters of a hypergroup. In $\$ 3$ we look at a restricted class of hypergroups, namely those on which there is a generalized translation in the sense of Levitan ([11], or see [12, p. 427]). (The notation of the present paper would seem to have two advantages over Levitan's: ours is compatible with current notation for compact groups, and in Levitan's notation, it is almost impossible to express correctly commutativity and associativity.) The theory for these hypergroups looks much like locally compact abelian group theory, yet covers a much wider range of examples. Finally in $\$ 4$ we discuss some examples and further questions.

1. Basic properties. We recall some standard notation (in the following, $X$ is a locally compact Hausdorff space):

Received by the editors January 13, 1972.

AMS (MOS) subject classifications (1970). Primary 22A20, 22A99, 43A10; Secondary 33A65, $42 \mathrm{~A} 60$.

Key words and phrases. Hypergroup, generalized translation operator, invariant measure, idempotent measure, central measures, characters, semigroup.

(') This research was partly supported by NSF grant GP-31483X. 
$C(X)$ : the space of complex continuous functions on $X$,

$C^{B}(X)$ : the space $\{f \in C(X): f$ bounded $\}$,

$C_{0}(X):$ the space $\left\{f \in C^{B}(X): f\right.$ tends to 0 at $\left.\infty\right\}$,

$C_{c}(X):$ the space $\left\{f \in C_{0}(X): f\right.$ has compact support $\}$,

$C_{c}^{+}(X):$ the set $\left\{f \in C_{c}(X): f \geq 0\right\}$,

$\|\cdot\|_{\infty}$ : the sup-norm on $C^{B}(X)$,

$M(X)$ : the space of finite regular Borel measures on $X$,

$M_{p}(X)$ : the set $\{\mu \in M(X): \mu \geq 0, \mu X=1\}$ (the probability measures),

spt $\mu$ : for $\mu \in M(X)$, the support of $\mu$, the maximum closed set $F \subset X$, such that $f \in C_{c}^{+}(X), f(x)>0$ for some $x \in F$ implies $\int_{X} f d|\mu|>0$,

$\delta_{x}$ : for $x \in X$, the unit point mass at $x$.

We will concern ourselves only with commutative hypergroups.

Definition 1.1. A locally compact space $H$ is called a hypergroup if there is a map $\lambda: H \times H \rightarrow M_{p}(H)$ with the following properties:

(1) $\lambda(x, y)=\lambda(y, x)(x, y \in H)$ (commutativity);

(2) for each $f \in C_{c}(H)$ the map $(x, y) \longmapsto \int_{H} f d \lambda(x, y)$ is in $C^{B}(H \times H)$, and $x \mapsto \int_{H} f d \lambda(x, y)$ is in $C_{c}(H)$ for each $y \in H$;

(3) the convolution on $M(H)$ defined implicitly by

$$
\begin{aligned}
& \int_{H} f d \mu * \nu=\int_{H} d \mu(x) \int_{H} d \nu(y) \int_{H} f d \lambda(x, y) \\
&\left(\mu, \nu \in M(H), f \in C_{0}(H)\right)
\end{aligned}
$$

is associative (note $\delta_{x} * \delta_{y}=\lambda(x, y)(x, y \in H)$ );

(4) (this property will not be needed in all of the theorems) there exists a (unique) point (the identity) $e \in H$ such that $\lambda(x, e)=\delta_{x}(x \in H)$.

Corollary 1.2. With the operations of addition and convolution, and with the measure norm, $M(H)$ is a commutative Banach algebra. Further it can be shown $M_{p}(H) * M_{p}(H) \subset M_{p}(H)$ (by extending formula (1-1) to $f \in C^{B}(H)$ ). The algebra $M(H)$ has an identity if and only if property 1.1(4) holds.

Defintion 1.3. For $A, B \subset H$ define $A \cdot B$ to be the closure of $\cup\{$ spt $\lambda(x, y): x$ $\in A, y \in B\}$.

Proposition 1.4. For $\mu, \nu \in M_{p}(H), \operatorname{spt}(\mu * \nu)=(\operatorname{spt} \mu) \cdot(\operatorname{spt} \nu)$.

Proof. Let $f \in C_{c}^{+}(H)$; then $\int_{H} f d \mu * \nu>0$ if and only if $\int_{H} f d \lambda(x, y)>0$ for some $(x, y) \in(\operatorname{spt} \mu) \times(\operatorname{spt} \nu)$, if and only if $f(z)>0$ for some $z \in$ spt $\lambda(x, y)$, some $(x, y) \in($ spt $\mu) \times($ spt $\nu)$, if and only if $f(z)>0$ for some $z \in$ $(\operatorname{spt} \mu) \cdot(\operatorname{spt} \nu)$.

Definition 1.5. Let $S$ be a closed subset of $H$. Say $S$ is a subhypergroup of $H$ if $x, y \in S$ implies spt $\lambda(x, y) \subset S$. This is equivalent to $S \cdot S \subset S$, and by Proposition 1.4, to $M(S)=\{\mu \in M(H):$ spt $\mu \subset S\}$ being a weak-* closed subalgebra of $M(H)$. 
Note that a subhypergroup is closed by definition. By analogy with the case of conjugacy classes of a compact group, we make the following definition.

Definition 1.6. The center of $H, Z(H)$, is the set $\{x \in H: \operatorname{spt} \lambda(x, y)$ is a single point for each $y \in H$ \}. For $x \in Z(H), y \in H$ defines $x \circ y \in H$ by $\lambda(x, y)$ $=\delta_{x \circ y}$.

Proposition 1.7. $Z(H)$ is a subhypergroup of $H$, and is itself a locally compact semigroup under the operation $\circ$.

Proof. By associativity $x, y \in Z(H)$ implies $x \circ y \in Z(H)$. Now suppose $\left\{x_{\alpha}\right\}$ is a net in $Z(H)$ with $x_{\alpha} \rightarrow_{\alpha} x \in H$. Fix $y \in H$ and let $f \in C_{c}^{+}(H)$ with $\int_{H} f d \lambda(x, y)>0$. Then $f\left(x_{\alpha} \circ y\right)=\int_{H} f d \lambda\left(x_{\alpha}, y\right) \rightarrow_{\alpha} \int_{H} f d \lambda(x, y)>0$. Thus for all large $\alpha, x_{\alpha} \circ y$ is in a fixed compact set (spt $f$ ). Let $z$ be a cluster point of $\left\{x_{\alpha} \circ y\right\}$; then $f(z)=\int_{H} f d \lambda(x, y)$ for all such $f$. This implies $\lambda(x, y)=\delta_{z}$ so $x \in Z(H)$.

Suppose $\left\{x_{\alpha}\right\},\left\{y_{\beta}\right\}$ are nets in $Z(H)$ with $x_{\alpha} \rightarrow_{\alpha} x, y_{\beta} \rightarrow_{\beta} y$. Then $\left(x_{\alpha}, y_{\beta}\right)$ $\rightarrow_{\alpha, \beta}(x, y)$ in $H \times H$, and for each $f \in C_{c}(H), f\left(x_{\alpha} \circ y_{\beta}\right)=\int_{H} f d \lambda\left(x_{\alpha}, y_{\beta}\right)$ $\rightarrow \int_{H} f d \lambda(x, y)=f(x \circ y)$, so the multiplication $\circ$ is jointly continuous.

Proposition 1.8. Let $H$ be a compact hypergroup (not necessarily with an identity). Then $M_{p}(H)$ is a commutative compact (jointly continuous) semigroup, under convolution and the weak-* topology (the topology $\sigma(M(H), C(H))$.

Proof. It is clear that $M_{p}(H)$ is weak-* compact and closed under convolution. Now let $\left\{\mu_{\alpha}\right\},\left\{\nu_{\beta}\right\}$ be nets in $M_{p}(H)$ with $\mu_{\alpha} \rightarrow_{\alpha} \mu, \nu_{\beta} \rightarrow_{\beta} \nu$ (weak-*), and let $f \in C(H)$. By the continuity of the map $(x, y) \longmapsto \int_{H} f d \lambda(x, y)$ and the StoneWeierstrass theorem there exist, for each $\varepsilon>0$, functions $g_{1}, \ldots, g_{n}, h_{1}, \ldots, h_{n}$ $\in C(H)$ such that

$$
\left|\int_{H} f d \lambda(x, y)-\sum_{j=1}^{n} g_{j}(x) h_{j}(y)\right|<\varepsilon \quad(x, y \in H) .
$$

Now

$$
\begin{aligned}
& \left|\int_{H} f d \mu_{\alpha} * \nu_{\beta}-\int_{H} f d \mu * \nu\right| \\
& \quad \leq 2 \varepsilon+\left|\sum_{j=1}^{n} \int_{H} g_{j} d \mu_{\alpha} \int_{H} h_{j} d \nu_{\beta}-\int_{H} g_{j} d \mu \int_{H} h_{j} d \nu\right| \\
& \quad \leq 2 \varepsilon+\sum_{j=1}^{n}\left\{\left\|h_{j}\right\|_{\infty}\left|\int_{H} g_{j} d\left(\mu_{\alpha}-\mu\right)\right|+\left\|g_{j}\right\|_{\infty}\left|\int_{H} h_{j} d\left(\nu_{\beta}-\nu\right)\right|\right\} .
\end{aligned}
$$

The last term can be made less than $4 \varepsilon$ by taking $\alpha$ and $\beta$ sufficiently large.

The proof of the above result followed the method of Rosenblatt [15]. We point out here that if $H$ is a compact topological space and $M(H)$ is a commutative Banach algebra under its natural norm, and a multiplication, denoted *, such that $M_{p}(H) * M_{p}(H) \subset M_{p}(H)$ and multiplication is jointly weak-* continuous on 
$M_{p}(H)$, then $H$ is a compact (commutative) hypergroup. Further the hypergroup convolution coincides with $*$. Here is a sketch of the argument: for $x, y \in H$ define $\lambda(x, y)=\delta_{x} * \delta_{y} \in M_{p}(H)$ and for $f \in C(H)$ define $T f(x, y)=\int_{H} f d \lambda(x, y)$. By assumption $T f$ is jointly continuous. The formula

$$
\int_{H} \int_{H} T f(x, y) d \mu(x) d \nu(y)=\int_{H} f d \mu * \nu \quad(f \in C(H))
$$

is first verified for finitely supported (discrete) measures in $M_{p}(H)$, and then is extended to all $\mu, \nu \in M_{p}(H)$ by weak-* continuity. (This involves a compactness argument similar to the one given in Theorem 1.10.)

Definition 1.9. For each $f \in C_{c}(H), x \in H, \mu \in M(H)$ define $R(x) f$ $\in C_{c}(H)$ by $R(x) f(y)=\int_{H} f d \lambda(y, x)(y \in H)$, and define the function $R(\mu) f$ by $R(\mu) f(y)=\int_{H} R(z) f(y) d \mu(z)(y \in H)$. (The following theorem shows that $R(\mu) f \in C_{0}(H)$.)

Note that commutativity implies $R(x) f(y)=R(y) f(x) \quad\left(f \in C_{c}(H), x, y\right.$ $\in H)$.

Theorem 1.10. For each $f \in C_{0}(H), \mu, \nu \in M(H)$, the function $R(\mu) f$ $\in C_{0}(H),\|R(\mu) f\|_{\infty} \leq\|\mu\|\|f\|_{\infty}$ and further $R(\nu)[R(\mu) f]=R(\nu * \mu) f$. Thus $R$ is a representation of $M(H)$ on $C_{0}(H)$.

Proof. By a uniform approximation argument and the regularity of measures in $M(H)$ it suffices to consider a fixed $f \in C_{c}(H)$ and a measure $\mu \in M_{p}(H)$ with compact support. Let $S=\operatorname{spt} \mu$ and let $K=\{R(x) f: x \in S\} \subset C_{0}(H)$. We claim $K$ is compact in the topology $\tau_{p}$ of pointwise convergence. In fact, let $\left\{R\left(x_{\alpha}\right) f\right\}$ be a net in $K$; then there exists a cluster point $x_{0} \in S$ of the set $\left\{x_{\alpha}\right\}$, so that for a cofinal subnet $R\left(x_{\beta}\right) f(y)=\int_{H} f d \lambda\left(y, x_{\beta}\right) \rightarrow_{\beta} \int_{H} f d \lambda\left(y, x_{0}\right)$ $=R\left(x_{0}\right) f(y) \quad(y \in H)$. By Grothendieck's compactness theorem [8, p. 182] $K$ is compact in the weak topology $\sigma\left(C\left(H_{\infty}\right), M\left(H_{\infty}\right)\right)$ (where $H_{\infty}$ is the one-point compactification of $H$ ). Now the norm-closed convex hull of $K, \overline{c o}(K)$, is weakly compact [3, p. 434], and thus $\tau_{p}$-compact. But $\mu$ is the weak-* limit of a net of discrete probability measures $\left\{\mu_{\alpha}\right\}$ on $S$, and for each $\alpha, R\left(\mu_{\alpha}\right) f \in \overline{\operatorname{co}}(K)$, so

$$
\begin{aligned}
R\left(\mu_{\alpha}\right) f(y) & =\int_{S} \int_{H} f d \lambda(y, z) d \mu_{\alpha}(z) \vec{\alpha} \int_{S} \int_{H} f d \lambda(y, z) d \mu(z) \\
& =R(\mu) f(y) \quad(y \in H) .
\end{aligned}
$$

Thus $R(\mu) f$ is in the $\tau_{p}$-closure of $\overline{\operatorname{co}}(K)$, but $\overline{c o}(K)$ is $\tau_{p}$-compact. Hence $R(\mu) f \in C_{0}(H)$ and $\|R(\mu) f\|_{\infty} \leq\|f\|_{\infty}\|\mu\|$.

Now let $f \in C_{0}(H), \mu, \nu \in M(H)$. For each $\sigma \in M(H)$ we have the identity $\int_{H} R(\mu) f d \sigma=\int_{H} f d \sigma * \mu$; thus

$$
\begin{aligned}
\int_{H} R(\nu)[R(\mu) f] d \sigma & =\int_{H} R(\mu) f d \sigma * \nu=\int_{H} f d((\sigma * \nu) * \mu) \\
& =\int_{H} f d(\sigma *(\nu * \mu))=\int_{H} R(\nu * \mu) f d \sigma .
\end{aligned}
$$


Observe that commutativity was not used in the above proof.

Definition 1.11. An invariant measure $m$ on $H$ is a positive nonzero regular Borel measure on $H$, which is finite on compact sets, and such that $\int_{H} f d m$ $=\int_{H} R(x) f d m\left(x \in H, f \in C_{c}(H)\right)$. If $m$ is finite, the latter condition can be written as $m * \delta_{x}=m(x \in H)$.

Theorem 1.12. Suppose $H$ is a compact hypergroup (not necessarily with an identity); then there exists a unique invariant measure $m_{H} \in M_{p}(\dot{H})$, and $m_{H}^{*} \mu$ $=m_{H} \quad\left(\mu \in M_{p}(H)\right)$. Further spt $m_{H}$ is the minimal closed ideal in $H$, that is, the smallest compact set $K$ such that $H \cdot K \subset K$.

Proof. The set $M_{p}(H)$ is weak-* compact and convex. Further $M_{p}(H)$ acts as a commutative semigroup of (weak-*) continuous linear operators on itself by convolution (see Proposition 1.8), so by the Markov-Kakutani theorem [3, p. 456] there exists $m_{H} \in M_{p}(H)$ such that $m_{H} * \mu=m_{H}$ for all $\mu \in M_{p}(H)$. In particular, $m_{H} * \delta_{x}=m_{H}(x \in H)$, that is, $\int_{H} R(x) f d m_{H}=\int_{H} f d m_{H}(x \in H, f \in$ $C(H))$. If $\mu \in M_{p}(H)$ such that $\mu * \delta_{x}=\mu(x \in H)$, then $\int_{H} f d \mu * m_{H}=$ $\int_{H} d m_{H}(x) \int_{H} R(x) f d \mu=\int_{H} f d \mu$, so $\mu * m_{H}=\mu$, but $\mu * m_{H}=m_{H}$ so $m_{H}=\mu$, proving uniqueness.

We observe that there exists a minimum closed ideal $I$ in $H$; in fact for any two nonempty closed ideals $I_{1}, I_{2}$, the set $I_{1} \cdot I_{2}$ is a closed ideal $\subset I_{1} \cap I_{2}$, and $H$ is compact. For $x \in I$ we have $m_{H}=\delta_{x} * m_{H}$ so, by Proposition 1.4, spt $m_{H}=\{x\} \cdot$ spt $m_{H} \subset I \cdot$ spt $m_{H} \subset I$. Conversely for any $x \in H,\{x\} \cdot$ spt $m_{H}$ $\subset$ spt $m_{H}$, so spt $m_{H}$ is a closed ideal $\subset I$, hence spt $m_{H}=I$.

The author knows of no general theorem about existence or uniqueness of an invariant measure for $H$ noncompact. In $\$ 3$ a uniqueness theorem is proved for *-hypergroups.

A measure $\mu$ is said to be idempotent if $\mu * \mu=\mu$. If $K$ is a compact subhypergroup of $H$, then the invariant measure $m_{K}$ of $K$ is idempotent, and $m_{K} \in M_{p}(H)$. In fact each idempotent probability measure arises this way; a fact discovered earlier for locally compact commutative semigroups by Glicksberg [7]. We have adapted some of his techniques in the following theorem.

Theorem 1.13. Let $\mu$ be idempotent and in $M_{p}(H)$. Then spt $\mu$ is a compact subhypergroup with no proper ideals, and $\mu$ is the invariant measure of $\operatorname{spt} \mu$.

Proof. Let $S=$ spt $\mu$. The steps of the proof are to show that $S$ is a subhypergroup, that $R(\mu) f$ is constant on the compact minimal ideal of $S$ for each $f \in C_{c}(H)$, that $S$ has no proper ideals, and finally that $\mu$ is invariant on $S$.

By Proposition 1.4, $S \cdot S=S$, so $S$ is a subhypergroup. We first observe that if $f \in C_{c}^{+}(H)$ and $f \neq 0$ on $S$ then $R(\mu) f(z)>0$ for some $z \in S$. Suppose not, then $\int_{H} \int_{H} f d \lambda(z, x) d \mu(x)=0$ for all $z \in S$, which implies $\int_{H} f d \lambda(z, x)=0$ for all $z, x \in S$ and $f=0$ on $S \cdot S=S$, a contradiction.

Let $f \in C_{c}^{+}(H), f \neq 0$ on $S$, and let $g=R(\mu) f \in C_{0}(H)$ by Theorem 1.10. Further $g \geq 0, g \neq 0$ on $S$, and $R(\mu) g=g$ since $R(\mu) g=R(\mu)[R(\mu) f]=R(\mu * \mu) f$ 
$=R(\mu) f=g$ (see 1.10). Since $g \in C_{0}(H)$, there exists $x_{0} \in S$ such that $g\left(x_{0}\right)=\|g\|_{S}(=\sup \{|g(x)|: x \in S\})$. But $g\left(x_{0}\right)=R(\mu) g\left(x_{0}\right)=\int_{S} R\left(x_{0}\right) g d \mu$ by commutativity. Now $R\left(x_{0}\right) g(y)=\int_{S} g d \lambda\left(y, x_{0}\right) \quad(y \in S)$, so $R\left(x_{0}\right) g(y)$ $\leq\|g\|_{s}$. Since $\mu \in M_{p}(S)$, this implies $R\left(x_{0}\right) g(y)=\|g\|_{S}$, for all $y \in S$. This implies that $g=\|g\|_{S}$ on $\left\{x_{0}\right\} \cdot S$, which is thus a compact ideal (since $g \in C_{0}(H)$ and $g \neq 0$ on $S$ ). Hence $S$ has a minimum compact nonempty ideal $I$ (see the proof of 1.12), so $I \subset\left\{x_{0}\right\} \cdot S$, and for each $f \in C_{c}^{+}(H), R(\mu) f$ is constant on $I$ with the value $\|R(\mu) f\|_{S}$.

We claim that $I=S$, for otherwise, there would exist $f \in C_{c}^{+}(H), f(z)>0$ some $z \in S$ and $f(I)=0$, but then $R(\mu) f(x)=\int_{S} \int_{S} f d \lambda(x, y) d \mu(y)=0$ for $x \in I$, since $x \in I, y \in S$ imply spt $\lambda(x, y) \subset I$, and this implies $\|R(\mu) f\|_{S}=0$, a contradiction.

To show that $\mu=m_{S}$ observe that $R(\mu) f\left(x^{\prime}\right)=R(\mu) f\left(x^{\prime \prime}\right) \quad\left(f \in C_{c}(H), x^{\prime}, x^{\prime \prime}\right.$ $\in S$ ) (note this was proved for $f \geq 0, f \not 0$ on $S$; if $f=0$ on $S$ then $R(\mu) f=0$ on $S$ ). Let $\mu^{\prime} \in M_{p}(S)$ so that $R(\mu) f(x)=\int_{S} f d \mu^{\prime}$, for all $f \in C_{c}(H)$ (note $R(\mu) f_{0} \mid S=1$ where $f_{0} \in C_{c}(H)$ with $\left.f_{0} \mid S=1\right)$ and so $\mu * \nu=\mu^{\prime}$ for all $\nu$ $\in M_{p}(S)$. (Recall $\int_{S} R(\mu) f d \nu=\int_{S} f d(\nu * \mu)$.) In particular $\mu^{\prime}=\mu * \mu=\mu$, so $\mu$ is invariant on $S$ and $\mu=m_{S}$.

2. The characters of a hypergroup. The idea of a character on a hypergroup encompasses characters of locally compact abelian groups, characters of compact nonabelian groups, and multiplication formulae for orthogonal polynomials.

Definition 2.1. A nonzero function $\phi \in C^{B}(H)$ is called a character if the following formula holds:

$$
\int_{H} \phi d \lambda(x, y)=\phi(x) \phi(y) \quad(x, y \in H) .
$$

The set of all characters will be denoted by $\hat{H}$. Observe that the constant function $1 \in \hat{H}$, and if $\phi \in \hat{H}$ then $\bar{\phi} \in \hat{H}$.

Proposition 2.2. For $\phi \in \hat{H}$, the following holds:

(1) $\phi(e)=1$.

(2) $|\phi(x)| \leq 1(x \in H)$.

(3) Let $G$ be a closed subgroup of the unit circle $\{z \in \mathbf{C}:|z|=1\}$ and let $S=\{x \in H: \phi(x) \in G\}$; then $S$ is a subhypergroup of $H$, and $\phi$ is constant with value $\phi(x) \phi(y)$ on spt $\lambda(x, y)$ for each $x, y \in S$. (Note the most interesting cases for $G$ are $\{1\}$ and $\{z:|z|=1\}$.)

(4) Suppose further that $|\phi(x)|=1(x \in H)$, then $\phi \psi \in \hat{H}$ for all $\psi \in \hat{H}$.

Proof. We have $\phi(e) \phi(x)=\phi(x)(x \in H)$ so $\phi(e)=1$. Let $M=\|\phi\|_{\infty}$. It follows from 2.1 that $M^{2} \leq M$, thus $M \leq 1$. If $x, y \in H$ such that $\phi(x), \phi(y)$ $\in G$, then $\left|\int_{H} \phi d \lambda(x, y)\right|=1$. But each point on the unit circle is an extreme point, so $\phi$ is constant with value $\phi(x) \phi(y) \in G$ on spt $\lambda(x, y)$. Hence spt $\lambda(x, y)$ $\subset S$. 
Suppose $|\phi(x)|=1(x \in H)$ and $\psi \in \hat{H}$. By (3) $\phi$ is constant on spt $\lambda(x, y)$ with value $\phi(x) \phi(y)$, for all $x, y \in H$. Now $\int_{H} \phi \psi d \lambda(x, y)=\phi(x) \phi(y) \int_{H} \psi d \lambda(x, y)$ $=(\phi \psi)(x)(\phi \psi)(y)(\psi \in \hat{H})$, so $\phi \psi \in \hat{H}$.

As expected, $\hat{H}$ can be considered as a subset of $\Delta$, the maximal ideal space of $M(H)$. In turn, $\Delta$ is viewed as a subset of $M(H)^{*}$, the dual space of $M(H)$.

Proposition 2.3. The set $\hat{H}$ may be identified with $\Delta \cap C^{B}(H)$. Indeed for each $\phi \in \hat{H}$, the map $\mu \longmapsto \overline{\hat{\mu}}(\phi)=\int_{H} \bar{\phi} d \mu(\mu \in M(H))$, is a multiplicative linear functional, and if $\phi \in C^{B}(H)$ such that $\mu \longmapsto \int_{H} \bar{\phi} d \mu$ is multiplicative, then $\phi \in \hat{H}$.

Proof. Let $\mu, \nu \in M(H)$ and extend formula (1-1) to bounded continuous functions. Then

$$
\begin{aligned}
(\mu * \nu)^{\wedge}(\phi) & =\int_{H} \bar{\phi} d \mu * \nu=\int_{H} \int_{H} \int_{H} \bar{\phi} d \lambda(x, y) d \mu(x) d \nu(y) \\
& =\int_{H} \bar{\phi} d \mu \int_{H} \bar{\phi} d \nu=\hat{\mu}(\phi) \hat{\nu}(\phi) \quad \text { for } \phi \in \hat{H}
\end{aligned}
$$

Conversely, if $\phi \in C^{B}(H)$ and $\mu \longmapsto \int_{H} \bar{\phi} d \mu$ is multiplicative, then $\bar{\phi}(x) \bar{\phi}(y)$ $=\int_{H} \bar{\phi} d \delta_{x} * \delta_{y}=\int_{H} \bar{\phi} d \lambda(x, y)(x, y \in H)$.

By analogy to the group case, one might hope for some dual structure on $\hat{H}$. As usual $\hat{H}$ is given the weak topology induced by the $\hat{\mu}, \mu \in M(H)$. It is not known to the author whether $\hat{H}$ is necessarily locally compact. However, if it is, let $\sigma \in M(\hat{H})$ and define $\mathcal{F}^{-1} \sigma(x)=\int_{H} \phi(x) d \sigma(\phi)(x \in H)$. Now one would like to define convolution on $\hat{H}$ so that it is transformed into pointwise multiplication on $H$. If this is to be a hypergroup structure, a necessary condition is that $\phi \psi$ be in the pointwise closure of the convex hull of $\hat{H}$ (in $C^{B}(H)$ ) for each $\phi, \psi \in \hat{H}$. We will later discuss cases where $\hat{H}$ is a hypergroup, and give an example of when it is not.

3. Hypergroups with involution. Up to this point we have not used any analogue of the group inverse, so the theorems presented so far work for semigroups as well as groups. However the examples we mentioned in the introduction do have more structure and we will now give the appropriate abstract definition. Levitan [11] first had the idea of generalizing locally compact groups by considering structures which under the analogue of the right regular representation are mapped onto selfadjoint sets of normal operators. These he called "generalized translation operators".

Definition 3.1. A locally compact hypergroup $H$ will be called a *-hypergroup if there exists an invariant measure $m$ on $H$ and a continuous involution $x \mapsto x^{\prime}(x \in H)$ such that

$$
\int_{H}(R(x) f) \bar{g} d m=\int_{H} f\left(R\left(x^{\prime}\right) g\right)^{-} d m
$$


( $\left.f, g \in C_{c}(H), x \in H\right)$, and such that $e \in \operatorname{spt} \lambda\left(x, x^{\prime}\right)(x \in H)$. The following properties are implicit in the word "involution": for each $\mu \in M(H)$ define $\mu^{\prime} \in M(H)$ by $\int_{H} f d \mu^{\prime}=\int_{H} f\left(x^{\prime}\right) d \mu(x)\left(f \in C_{c}(H)\right)$, then $\mu \longmapsto \mu^{\prime}$ is an involution on $M(H)$ and $\lambda\left(x^{\prime}, y^{\prime}\right)=\lambda(y, x)^{\prime}(x, y \in H)$.

For a function $f$ on $H$, the function $f^{\prime}$ is defined by $f^{\prime}(x)=f\left(x^{\prime}\right)(x \in H)$. For a regular Borel, finite on compact sets, measure $\mu$ on $H, \mu^{\prime}$ is defined by $\int_{H} f d \mu^{\prime}=\int_{H} f^{\prime} d \mu\left(f \in C_{c}(H)\right)$.

In the example of the space of conjugacy classes of a compact group, the involution maps the conjugacy class of a point to the conjugacy class of the point's inverse (see Example 4.1 for more details).

Proposition 3.2. The invariant measure $m$ of a *-hypergroup has the following properties:

(1) the support of $m$ is $H$; that is, if $V$ is open in $H$ and $V \neq \varnothing$ then $m V>0$;

(2) $m$ is unique up to a constant; that is, if $m_{1}$ is an invariant measure on $H$ then $m_{1}=c m$ for some $c>0$;

(3) $m=m^{\prime}$.

Proof. To prove (1) let $x \in$ spt $m$ and let $f \in C_{c}^{+}(H)$ with $f(e)>0$. Now $R\left(x^{\prime}\right) f(x)=\int_{H} f d \lambda\left(x, x^{\prime}\right)>0$ (since $\left.e \in \operatorname{spt} \lambda\left(x, x^{\prime}\right)\right)$ so $\int_{H} R\left(x^{\prime}\right) f d m>0$. By invariance, $\int_{H} f d m=\int_{H} R\left(x^{\prime}\right) f d m>0$, hence $e \in$ spt $m$. For any $y \in H$, let $f \in C_{c}^{+}(H)$ with $f(y)>0$, and thus $R(y) f(e)=f(y)>0$. Now $\int_{H} f d m$ $=\int_{H} R(y) f d m>0$, which implies that $y \in$ spt $m$.

As a first step in proving (2) and (3) we show that if $m_{1}$ is an invariant measure on $H$, then so is $m_{1}^{\prime}$. Observe that $R(x) f\left(y^{\prime}\right)=\int_{H} f d \lambda\left(y^{\prime}, x\right)=\int_{H} f^{\prime} d \lambda\left(x^{\prime}, y\right)$ $=R\left(x^{\prime}\right) f^{\prime}(y)$ (using commutativity) $\left(f \in C_{c}(H), x, y \in H\right)$. Let $m_{1}$ be an invariant measure on $H$. Then $\int_{H}(R(x) f) d m_{1}^{\prime}=\int_{H} R(x) f\left(y^{\prime}\right) d m_{1}(y)=$ $\int_{H} R\left(x^{\prime}\right) f^{\prime}(y) d m_{1}(y)=\int_{H} f^{\prime}(y) d m_{1}(y)$ (by invariance) $=\int_{H} f d m_{1}^{\prime}$ for $f \in$ $C_{c}(H), x \in H$. Thus $m_{1}^{\prime}$ is also invariant.

Let $f, g \in C_{c}^{+}(H)$. Observe that the functions $(x, y) \longmapsto R(x) f(y) g(y)$ and $(x, y) \longmapsto f(y) R\left(x^{\prime}\right) g(y)$ are in $C^{B}(H \times H)$, and hence are Borel measurable. Further the integrals

$$
\int_{H} \int_{H} R(x) f(y) g(y) d m(y) d m_{1}(x) \text { and } \int_{H} \int_{H} f(y) R\left(x^{\prime}\right) g(y) d m(y) d m_{1}(x)
$$

are finite and equal. They are equal because the inner integrals are equal functions of $x$ by Definition 3.1. In fact, by Fubini's theorem, the first integral equals

$$
\int_{H} \int_{H} g(y) R(y) f(x) d m_{1}(x) d m(y)=\int_{H} \int_{H} g(y) f(x) d m_{1}(x) d m(y)
$$

(by invariance of $m_{1}$ )

$$
=\left(\int_{H} g d m\right)\left(\int_{H} f d m_{1}\right)<\infty
$$


The second integral equals

$$
\begin{aligned}
\int_{H} \int_{H} f(y) R(y) g\left(x^{\prime}\right) d m_{1}(x) d m(y) & =\int_{H} \int_{H} f(y) R(y) g(x) d m_{1}^{\prime}(x) d m(y) \\
& =\int_{H} f d m \int_{H} g d m_{1}^{\prime}
\end{aligned}
$$

(by the invariance of $m_{1}^{\prime}$ ). Since $f$ and $g$ are arbitrary, the relation $\int_{H} f d m \int_{H} g d m_{1}^{\prime}=\int_{H} g d m \int_{H} f d m_{1}$ implies there exists a constant $c>0$ such that $\int_{H} f d m_{1}=\int_{H} f d m_{1}^{\prime}=c \int_{H} f d m$, for all $f \in C_{c}(H)$. This proves (2) and (3).

Observe that (1) of Proposition 3.2 shows that $H$ has no proper closed ideals.

Proposition 3.3. The center $Z(H)$ of $a^{*}$-hypergroup $H$ is a closed subgroup, with identity e.

Proof. By Proposition 1.7, $Z(H)$ is a closed subsemigroup. Let $x \in Z(H)$, $y \in H$; then $\lambda\left(x^{\prime}, y\right)=\lambda\left(y^{\prime}, x\right)^{\prime}=\left(\delta_{x \circ y^{\prime}}\right)^{\prime}=\delta_{\left(x \circ y^{\prime}\right)^{\prime}}$, so $x^{\prime} \in Z(H)$. Also $e$ $\in \operatorname{spt} \lambda\left(x, x^{\prime}\right)=\left\{x \circ x^{\prime}\right\}$, so $x^{\prime}$ is the group inverse of $x \in Z(H)$.

Proposition 3.4. The space $L^{1}(H)$, that is, $L^{1}(m)$, is a closed ideal in $M(H)$, and for $f \in C_{c}(H), \mu \in M(H)$, the following formula holds:

$$
f * \mu(x)=\int_{H} R(x) f\left(y^{\prime}\right) d \mu(y)(x \in H), \text { and } f * \mu \in C_{0}(H) \cap L^{1}(H),
$$

where convolution is to be interpreted as the measure convolution of $\mu$ with $f \cdot m$ (the measure $f d m$ ).

Proof. Let $g \in C_{c}(H)$ and let $\nu=\mu *(f \cdot m)$; then

$$
\begin{aligned}
\int_{H} g d \nu & =\int_{H} d \mu(y) \int_{H} f(x) d m(x) \int_{H} g d \lambda(y, x) \\
& =\int_{H} d \mu(y) \int_{H} f(x)(R(y) g(x)) d m(x) \\
& =\int_{H} d \mu(y) \int_{H}\left(R\left(y^{\prime}\right) f(x)\right) g(x) d m(x) \\
& =\int_{H} g(x) d m(x) \int_{H} R(x) f\left(y^{\prime}\right) d \mu(y) \quad \text { (Fubini's theorem). }
\end{aligned}
$$

Observe that $\int_{H} R(x) f\left(y^{\prime}\right) d \mu(y)=R\left(\mu^{\prime}\right) f(x)$ so this function is in $C_{0}(H)$, by Theorem 1.10. The inequality $\left|\int_{H} g d \nu\right| \leq\|g\|_{\infty}\|\mu\|\|f\|$ shows that $R\left(\mu^{\prime}\right) f$ is in $L^{1}(H)$. The density of $C_{c}(H)$ in $L^{1}(H)$ finishes the proof.

Levitan observed that $\hat{H}$ can be identified with the maximal ideal space of $L^{1}(H)$ and that $L^{1}(H)$ is semisimple. He also observed that there exists a regular Borel measure on $\hat{H}$ so that there is a Plancherel formula for $L^{2}(H)$ (see [12, p. 428] for a sketch of the proofs of these results). We will only use this fact for the case where $H$ is compact. 
Henceforth $H$ will be a compact *-hypergroup. Hence there is a unique invariant measure $m$ with $m H=1$ and, further, spt $m=H$.

Theorem 3.5. The set $\hat{H}$ is an orthogonal basis for $L^{2}(H)$ (that is, $\left.L^{2}(m)\right)$. Further $\phi\left(x^{\prime}\right)=\overline{\phi(x)}$ for $x \in H, \phi \in \hat{H}$.

Proof. From Levitan's Plancherel theorem [12, p. 429] we have the density of the span of $\hat{H}$. (This theorem can be directly proved by mimicking the compact group argument using compact operator theory; see for example [5, p. 74].) Let $\phi, \psi \in \hat{H}, x \in H$, then $\int_{H}(R(x) \phi) \bar{\psi} d m=\phi(x) \int_{H} \phi \bar{\psi} d m=\int_{H} \phi\left(R\left(x^{\prime}\right) \psi\right)^{-} d m$ $=\overline{\psi\left(x^{\prime}\right)} \int_{H} \phi \bar{\psi} d m$. Thus $\left(\phi(x)-\overline{\left.\psi\left(x^{\prime}\right)\right)} \int_{H} \phi \bar{\psi} d m=0\right.$. First let $\psi=\phi$, then $\phi(e)$ $=1$, so $\int_{H}|\phi|^{2} d m>0$, so $\phi\left(x^{\prime}\right)=\overline{\phi(x)}$. Then let $\phi \neq \psi$ and so $\int_{H} \phi \bar{\psi} d m=0$.

This theorem shows that $\hat{H}$ is discrete in the weak-* topology.

Definition 3.6. For $\phi \in \hat{H}$, let $c(\phi)=\left(\int_{H}|\phi|^{2} d m\right)^{-1} \geq 1$. Thus each $f$ $\in L^{2}(H)$ has the Fourier series $\sum_{\phi \in A} c(\phi) \hat{f}(\phi) \phi$, where $\hat{f}(\phi)=\int_{H} f \bar{\phi} d m$ (see 2.3), and the Plancherel theorem says $\|f\|_{2}=\left(\sum_{\phi} c(\phi)|\hat{f}(\phi)|^{2}\right)^{1 / 2}$.

The group case leads us to expect the following sort of theorem.

Theorem 3.7. For each $x \in H, R(x)$ extends to a bounded operator on $L^{2}(H)$, and the map $x \longmapsto R(x) f$ is continuous for each $f \in L^{2}(H)$. Further $R(x)^{*}=R\left(x^{\prime}\right)$ (where * denotes the operator adjoint), and $(R(x) f)^{\wedge}(\phi)=\phi(x) \hat{f}(\phi)(x \in H, f$ $\left.\in L^{2}(H), \phi \in \hat{H}\right)$.

Proof. Let $f \in C(H), \phi \in \hat{H}, x \in H$, then $(R(x) f)^{\wedge}(\phi)=\int_{H} R(x) f \bar{\phi} d m$ $=\int_{H} f\left(R\left(x^{\prime}\right) \phi\right)^{-} d m=\phi(x) \int_{H} f \bar{\phi} d m=\phi(x) \hat{f}(\phi)$. Clearly the operator $R(x)$ extends to a bounded operator on $L^{2}(H)$ with operator norm equal to $\sup \{|\phi(x)|: \phi$ $\in \hat{H}\} \leq 1$. The rest of the proof is straightforward.

The conditions for $H$ to be a *-hypergroup do not seem to imply that $\mathrm{sp} \hat{H}$ is sup-norm dense in $C(H)$ (however the author knows of no counterexample). In the particular cases of interest there holds a further property:

$$
\hat{H} \hat{H} \subset \operatorname{co}(\hat{H}),
$$

that is, for each $\phi, \psi \in H$, there exists a nonnegative real function $n(\phi, \psi ; \omega)$ on $\hat{H}$ with only finitely many nonzero values, such that

$$
\phi(x) \psi(x)=\sum_{\omega \in H} n(\phi, \psi ; \omega) \omega(x) \quad(x \in H)
$$

(setting $x=e$ this implies $\sum_{\omega \in A} n(\phi, \psi ; \omega)=1$ ).

If $H$ has this additional property, we will call it a $\mathrm{P}^{*}$-hypergroup. A *hypergroup need not have property (P); see Example 4.8. Henceforth $H$ will be $a$ compact $\mathrm{P}^{*}$-hypergroup.

Proposition 3.8. The span of $\hat{H}$ is sup-norm dense in $C(H)$.

Proof. Indeed the linear span of $\hat{H}$ is a selfadjoint algebra containing the constants. Further for $x \neq y, x, y \in H$, let $f \in C(H)$ with $f(x) \neq f(y)$. Then 
$R(x) f(e)=f(x) \neq f(y)=R(y) f(e)$; thus $R(x) f \neq R(y) f$ in $L^{2}(H)$. By Theorem 3.7 this implies that some $\phi \in A$ separates $x$ and $y$. The Stone-Weierstrass theorem finishes the proof.

We interpret $M(\hat{H})=l^{1}(\hat{H})$ as the space of absolutely convergent Fourier series on $H$, that is, for $\mu \in M(\hat{H})$ write $\mathcal{F}^{-1} \mu(x)=\sum_{\phi \in A} \mu(\phi) \phi(x)$, where $\|\mu\|=\sum_{\phi}|\mu(\phi)|$. Property (P) implies that $\hat{H}$ is a discrete hypergroup where the convolution is defined so that $\mathcal{Y}^{-1}$ is multiplicative and the function 1 is the identity. In particular the product of the unit masses at $\phi, \psi \in \hat{H}$ is the probability measure $n(\phi, \psi)$. Observe that there is on hand a separating collection of multiplicative linear functionals on $M(\hat{H})$, namely, the maps $\mu$ $\longmapsto \mathcal{P}^{-1} \mu(x)(x \in H)$. We will not concern ourselves with the question of whether the maximal ideal space of $M(\hat{H})$ is $H$; see however Levitan [12, p. 429].

For $f \in C_{c}(H)(\phi \in \hat{H}), R(\phi) f$ is given by $R(\phi) f(\psi)=\sum_{\omega \in A} f(\omega) n(\psi, \phi ; \omega)$.

Theorem 3.9. The measure $c$ on $\hat{H}$ is invariant, and with $\phi \longmapsto \bar{\phi}$ as the involution, $\hat{H}$ is $a^{*}$-hypergroup.

Property (3-1) in terms of $c$ is

$$
\begin{aligned}
\sum_{\omega \in H}(R(\phi) f)(\omega) \bar{g}(\omega) c(\omega)=\sum_{\omega \in A} f(\omega)(R(\phi) g(\omega))^{-} c(\omega) & \\
& \left(f, g \in C_{c}(\hat{H}), \phi \in H\right) .
\end{aligned}
$$

$\left(\right.$ Recall $c(\phi)=\left(\int_{H}|\phi|^{2} d m\right)^{-1}$. $)$

Proof. Observe that $\phi \psi=\sum_{\omega \in A} n(\phi, \psi ; \omega) \omega$ and Theorem 3.5 show that

$$
n(\phi, \psi ; \omega)=c(\omega) \int_{H} \phi \psi \bar{\omega} d m
$$

Let $f \in C_{c}(\hat{H}), \phi \in \hat{H}$, then

$$
\begin{aligned}
\sum_{A} c R(\phi) f & =\sum_{\omega} c(\omega)(R(\phi) f)(\omega) \\
& =\sum_{\omega} c(\omega) \sum_{\psi} f(\psi) n(\phi, \omega ; \psi),
\end{aligned}
$$

but

$$
\begin{aligned}
c(\omega) n(\phi, \omega ; \psi) & =c(\omega) c(\psi) \int_{H} \phi \omega \bar{\psi} d m \\
& =c(\omega) c(\psi) n(\bar{\phi}, \psi ; \omega) / c(\omega) \\
& =c(\psi) n(\bar{\phi}, \psi ; \omega) .
\end{aligned}
$$

Thus

$$
\begin{aligned}
\sum_{A} c R(\phi) f & =\sum_{\psi} f(\psi) \sum_{\omega} c(\psi) n(\Phi, \psi ; \omega) \\
& =\sum_{\psi} c(\psi) f(\psi)=\sum_{A} c f
\end{aligned}
$$


so $c$ is invariant. Now let $f, g \in C_{c}(\hat{H}), \phi \in \hat{H}$, then

$$
\begin{aligned}
\sum_{A} c(R(\phi) f) \bar{g} & =\sum_{\omega} c(\omega) \bar{g}(\omega) \sum_{\psi} f(\psi) n(\phi, \omega ; \psi) \\
& =\sum_{\psi} f(\psi) \sum_{\omega} c(\psi) \bar{g}(\omega) n(\phi, \psi ; \omega) \\
& =\sum_{\psi} c(\psi) f(\psi)((R(\phi) g)(\psi))^{-} \\
& =\sum_{A} c f(R(\phi) g)^{-}
\end{aligned}
$$

Also $n(\phi, \bar{\phi} ; 1)=\int_{H}|\phi|^{2} d m>0$; thus $1 \in$ spt $n(\phi, \bar{\phi})$, and so $\hat{H}$ is a *-hypergroup.

Corollary 3.10. $Z(H)=\{\phi \in A:|\phi(x)|=1(x \in H)\}=\{\phi \in H: c(\phi)=1\}$, and $Z(A)$ is a discrete abelian group.

Proof. (4) of Proposition 2.2 shows that $\{\phi \in A:|\phi| \equiv 1\} \subset Z(\hat{H})$. Conversely, let $\phi \in Z(A)$, then $\phi \bar{\phi} \in A$, but $\phi \bar{\phi}$ is not orthogonal to 1 , so $\phi \bar{\phi}=1$ by 3.5. Clearly $|\phi| \equiv 1$ if and only if $c(\phi)^{-1}=\int_{H}|\phi|^{2} d m=1$.

Definition 3.11. A subhypergroup $S$ of $H$ (or $\hat{H}$ ) is said to be normal if $x \in S$ (or $\phi \in S$ ) implies $x^{\prime} \in S$ (or $\bar{\phi} \in S$ ). For $E \subset A$, let $E^{\perp}=\{x \in H: \phi(x)$ $=1(\phi \in E)\}$, then $E^{\perp}$ is a normal subhypergroup of $H$. For $F \subset H$, let $F^{\perp}=\{\phi \in \hat{H}: \phi(x)=1(x \in F)\}$, then $F^{\perp}$ is a normal subhypergroup of $\hat{H}$. These facts are clear from Proposition 2.2.

Proposition 3.12. Let $F=\bigcup\left\{\right.$ spt $\left.\lambda\left(x, x^{\prime}\right): x \in H\right\}$; then $F^{i}=Z(A)$.

Proof. Indeed $\phi \in Z(H)$ if and only if $1=|\phi(x)|^{2}=\phi(x) \phi\left(x^{\prime}\right)=\int_{H} \phi d \lambda\left(x, x^{\prime}\right)$ $(x \in H)$, if and only if $\phi=1$ on spt $\lambda\left(x, x^{\prime}\right)$ for all $x \in H$.

Proposition 3.13. $Z(H)=\{x \in H:|\phi(x)|=1(\phi \in A)\}=E^{\perp}$, where $E=$ $\cup\{$ spt $n(\phi, \bar{\phi}): \phi \in \hat{H}\}$.

Proof. If $x \in Z(H)$, then $|\phi(x)|^{2}=\phi(x) \phi\left(x^{\prime}\right)=\int_{H} \phi d \lambda\left(x, x^{\prime}\right)=\phi\left(x \circ x^{\prime}\right)$ $=\phi(e)=1$. Now suppose $x \in H$ with $|\phi(x)|=1$ for all $\phi \in A$. For any $\phi, \psi \in A$ we have $1=|\phi(x) \psi(x)|=\left|\sum_{\omega \in A} n(\phi, \psi ; \omega) \omega(x)\right|$, and by convexity, $\omega(x)=\phi(x) \psi(x)$ for all $\omega \in \operatorname{spt} n(\phi, \psi)$. For each $y \in H$ the map $\mu$ $\longmapsto \int_{H} \mathcal{\Im}^{-1} \mu d \lambda(x, y)$ is multiplicative on $M(A)$. In fact for $\phi, \psi \in A$,

$$
\begin{aligned}
\int_{H} \phi \psi d \lambda(x, y) & =\sum_{\omega} n(\phi, \psi ; \omega) \int_{H} \omega d \lambda(x, y)=\sum_{\omega} n(\phi, \psi ; \omega) \omega(x) \omega(y) \\
& =\phi(x) \psi(x) \sum_{\omega} n(\phi, \psi ; \omega) \omega(y)=\phi(x) \psi(x) \phi(y) \psi(y) \\
& =\left(\int_{H} \phi d \lambda(x, y)\right)\left(\int_{H} \psi d \lambda(x, y)\right) .
\end{aligned}
$$

From the density of $\left\{\mathcal{Y}^{-1} \mu: \mu \in M(\hat{H})\right\}$ in $C(H)$ it follows that $\lambda(x, y)=\delta_{z}$, some $z \in H$. Hence $x \in Z(H)$. Finally for $x \in H, x \in Z(H)$ if and only if $1=|\phi(x)|^{2}=\phi(x) \bar{\phi}(x)=\sum_{\psi} n(\phi, \bar{\phi} ; \psi) \psi(x)(\phi \in A)$, if and only if $\psi(x)=1$ for 
all $\psi \in \operatorname{spt} n(\phi, \bar{\phi})$, some $\phi \in \hat{H}$.

There is a classical theroem which asserts that any closed subsemigroup of a compact group is a subgroup. The analogue holds for compact $\mathrm{P}^{*}$-hypergroups.

Theorem 3.14. Let $K$ be a (nonempty) (closed) subhypergroup of $H$; then $K$ is normal.

Proof. By Theorem 1.12 there exists an invariant measure $m_{K}$ on $K$, and spt $m_{K}$ is the minimum closed ideal $I$ in $K$. For $\phi \in I^{\perp},\left(m_{K}\right)^{\wedge}(\phi)=\int_{I} \bar{\phi} d m_{K}=1$. For $\phi \notin I^{\perp}$, let $x \in I$ with $\phi(x) \neq 1$; then $\left(m_{K}\right)^{\wedge}(\phi)=\int_{I} \bar{\phi} d m_{K}=\int_{I}(R(x) \phi)^{-} d m_{K}$ $=\overline{\phi(x)} \int_{I} \bar{\phi} d m_{K}=\overline{\phi(x)}\left(m_{K}\right)^{\wedge}(\phi)$, so $\left(m_{K}\right)^{\wedge}(\phi)=0$ (the second equality comes from the invariance of $m_{K}$ ). We claim that $I$ is a normal subhypergroup of $H$. Indeed, let $f \in C(H)$ be of the form $\sum_{\phi} a(\phi) \phi(a(\phi) \in \mathbf{C}$, all but finitely many $a(\phi)$ equal 0 ); then $\int_{H} f d m_{K}^{\prime}=\int_{H} f d m_{K}$ (since $\int_{H} \phi\left(x^{\prime}\right) d m_{K}(x)=\left(m_{K}\right)^{\wedge}(\phi)$ $=\left(m_{K}\right)^{\wedge}(\bar{\phi})=\int_{H} \phi d m_{K}$ for all $\left.\phi \in H\right)$. The set of such $f$ is dense in $C(H)$, so $m_{K}=m_{K}^{\prime}$. Thus $x \in I$ implies $x^{\prime} \in I$. We conclude that $e \in I$, but $I$ is an ideal in $K$, so $K=I$, a normal subhypergroup.

We will now show that any (closed) subhypergroup $K$ of $H$ is itself a $\mathrm{P}^{*}$. hypergroup, and $\hat{K}$ may be identified with a hypercoset structure on $\hat{H}$. A hypercoset is a subset of $\hat{H}$ of the form $\phi \cdot E$, where $\phi \in \hat{H}$ and $E$ is a subhypergroup of $\hat{H}$.

Definition 3.15. Let $\rho$ be the restriction map $C(H) \rightarrow C(K)$. Observe $\rho \hat{H} \subset R$. Define an equivalence relation on $\hat{H}$ by $\phi \sim \psi$ if and only if $\rho \phi=\rho \psi(\phi, \psi$ $\in \hat{H})$.

Proposition 3.16. For $\phi, \psi \in \hat{H}, \rho \phi=\rho \psi$ if and only if $\phi \in \psi \cdot K^{\perp}$, that is, there exists $\omega \in K^{\perp}$ such that $n(\psi, \omega ; \phi)>0$. Thus the equivalence classes of $\sim$ are exactly the hypercosets $\psi \cdot K^{\perp}$.

Proof. For any $\phi \in \hat{H}, \int_{K} \phi d m_{K}=0$, unless $\phi \in K^{\perp}$, that is, unless $\rho \phi=1$ (recall $m_{K}$ is the invariant measure on $K$ and see Theorem 3.14). Let $\phi, \psi \in A$ with $\rho \phi=\rho \psi$, then $\int_{K} \rho(\phi \bar{\psi}) d m_{K}>0$ (since spt $m_{K}=K$ by 3.14). But $\phi \bar{\psi}$ $=\sum_{\omega} n(\phi, \bar{\psi} ; \omega) \omega$, so $\int_{K} \rho(\phi \bar{\psi}) d m_{K}=\sum_{\rho \omega=1} n(\phi, \bar{\psi} ; \omega)>0$. Thus there exists $\omega$ $\in K^{\perp} \quad$ such that $n(\phi, \bar{\psi} ; \omega)>0$, but $n(\phi, \bar{\psi} ; \omega)=c(\omega) \int_{H} \phi \bar{\psi} \bar{\omega} d m$ $=c(\omega) \int_{H} \psi \omega \bar{\phi} d m=c(\omega) n(\psi, \omega ; \phi) / c(\phi)$, so $\phi \in \psi \cdot K^{\perp}$.

Conversely if $\phi \in \psi \cdot K^{\perp}$, then there exists $\omega \in K^{\perp}$ so that $n(\psi, \omega ; \phi)>0$. Thus $\rho \psi=\rho(\psi \omega)=\sum_{\rho \chi=\rho \phi} n(\psi, \omega ; \chi) \rho \phi+\sum_{\rho \chi \neq \rho \phi} n(\psi, \omega ; \chi) \rho \chi$. But characters (on $K)$ are linearly independent (consider them as multiplicative linear functionals on $M(K))$ so $\sum_{\rho \chi=\rho \phi} n(\psi, \omega ; \chi)=1$, and $n(\psi, \omega ; \chi)=0$ for $\rho \chi \neq \rho \phi$. Thus $\rho \psi=\rho \phi$.

Theorem 3.17. Let $K$ be a subhypergroup of $H$; then $K$ is a $\mathrm{P}^{*}$-hypergroup, and the restriction map takes $\hat{H}$ onto $\hat{K}$. Further $\hat{K}$ may be identified with the space of hypercosets of $K^{\perp}$.

Proof. We first claim for $\phi, \psi \in \hat{H}$ with $\rho \phi \neq \rho \psi$ that $\int_{K}(\rho \phi)(\rho \psi)^{-} d m_{K}=0$. In fact 


$$
\begin{aligned}
\int_{K}(\rho \phi)(\rho \psi)^{-} d m_{K} & =\sum_{\omega \in A} n(\phi, \bar{\psi} ; \omega) \int_{K}(\rho \omega) d m_{K} \\
& =\sum_{\omega \in K^{\perp}} n(\phi, \bar{\psi} ; \omega) .
\end{aligned}
$$

By Proposition 3.16, each $n(\phi, \bar{\psi} ; \omega)\left(\omega \in K^{\perp}\right)$ is zero (or else $\phi \in \psi \cdot K^{\perp}$ ).

We already have an involution and an invariant measure on $K$, so we need to show $\int_{K} R(x) f \bar{g} d m_{K}=\int_{K} f\left(R\left(x^{\prime}\right) g\right)^{-} d m_{K}$, for $x \in K, f, g \in C(K)$. It will suffice to prove this formula for restrictions of finite linear combinations of $\hat{H}$, since these are dense in $C(K)$. Let $f=\sum_{\phi} a(\phi) \rho \phi, g=\sum_{\psi} b(\psi) \rho \psi$ (finite sums, with no repetitions, that is, no more than one representative from each hypercoset), and let $x \in K$. Then

$$
\begin{aligned}
\int_{K} R(x) f \bar{g} d m_{K} & =\sum_{\phi, \psi} a(\phi) \overline{b(\psi)} \int_{K}(R(x) \rho \phi)(\rho \psi)^{-} d m_{K} \\
& =\sum_{\phi, \psi} a(\phi) \overline{b(\psi)} \phi(x) \int_{K}(\rho \phi)(\rho \psi)^{-} d m_{K} \\
& =\sum_{\phi} a(\phi) \overline{b(\phi)} \phi(x) c(\rho \phi)^{-1} \\
& =\sum_{\phi} a(\phi) \overline{b(\phi) \phi\left(x^{\prime}\right) c(\rho \phi)^{-1}} \\
& =\int_{K} f\left(R\left(x^{\prime}\right) g^{-} d m_{K} .\right.
\end{aligned}
$$

Thus $K$ is a *-hypergroup, and we see that $\rho H$ is an orthogonal basis for $L^{2}(K)$, hence $\rho \hat{H}=R$. To see that $K$ has property $(\mathrm{P})$, observe that $R \hat{K}=(\rho \hat{H})(\rho \hat{H})$ $\subset \rho(\operatorname{co}(\hat{H}))=\operatorname{co}(\rho \hat{H})=\operatorname{co}(\hat{R})$.

We now consider the problem of idempotent measures. Note $M(H)$ is semisimple by Propositions 2.3 and 3.8. For each idempotent $\mu \in M(H)$ (that is, $\hat{\mu}(\phi)=1$ or 0 , all $\phi \in \hat{H}$ ) let $S(\mu)=\{\phi \in \hat{H}: \hat{\mu}(\phi)=1\}$. As usual we see that $g=\{S(\mu) \subset A: \mu$ idempotent $\}$ is closed under complementation, finite unions and finite intersection. We will show that $I$ contains all hypercosets. Rider [14] has done this for central measures on compact groups.

Theorem 3.18. Suppose $H$ is a compact $\mathrm{P}^{*}$-hypergroup and $K$ is a subhypergroup. Then each hypercoset of $K^{\perp}$ is $S(\mu)$ for some idempotent $\mu \in M(H)$.

Proof. By Proposition 3.16 and Theorem $3.17, K$ is normal and is itself $\mathrm{P}^{*}$. Let $E$ be a hypercoset of $K^{\perp}$, that is, $E=\psi \cdot K^{\perp}$ for some $\psi \in \hat{H}$. Now let $d \mu=c \psi d m_{K}$, where $c=\left(\int_{K}|\psi|^{2} d m_{K}\right)^{-1}$. Then for $\phi \in \hat{H}, \hat{\mu}(\phi)=c \int_{K} \psi \bar{\phi} d m_{K}$ which is zero for $\phi|K \neq \psi| K$, that is, $\phi \notin \psi \cdot K^{\perp}$ (by Proposition 3.16), and $\hat{\mu}(\phi)=1$ for $\phi|K=\psi| K$. Hence $S(\mu)=E$.

4. Examples and remarks. We will use [5, Chapters 7, 9] as a reference for the notation and some theorems on compact groups and compact homogeneous spaces.

Example 4.1. Let $G$ be a compact nonabelian group with normalized Haar measure $m_{G}$. Let $H$ be the space of conjugacy classes of $G$; then $M(H)$ can be 
identified with $\mathcal{Z M}(G)$, the center of $M(G)$. The point masses of $H$ correspond to measures of the form $f \longmapsto \int_{G} f\left(y x y^{-1}\right) d m_{G}(y)(f \in C(G)$, fixed $x \in G)$. Under the correspondence $\mathcal{Z} M(G) \rightarrow M(H), m_{G}$ goes over to the invariant measure on $H$. The involution on $H$ is $\left\{y x y^{-1}: y \in G\right\}^{\prime}=\left\{y x^{-1} y^{-1}: y \in G\right\}$. The center of $H, Z(H)$, consists of the one-point conjugacy classes of $G$, namely the center of $G$. From the remark after Theorem 1.8 we see that $H$ is a ${ }^{*}$-hypergroup. The characters of $H$ are the function $\left\{\chi_{\alpha} / n_{\alpha}: \alpha \in \hat{G}\right\}$ ( $\chi_{\alpha}$ is a character of $G, n_{\alpha}$ is its degree [5, p. 77]). From the formula $\chi_{\alpha} \chi_{\beta}=\sum_{\gamma} m_{\alpha \beta}(\gamma) \chi_{\gamma}(\alpha, \beta \in \hat{G})$ (see [5, p. 79]), $m_{\alpha \beta}(\gamma) \geq 0$, we see that $H$ is a $\mathrm{P}^{*}$-hypergroup.

Dičman [2] has considered conjugacy classes from this point of view. See also the work of Ragozin [13] on the central measure algebra of a compact Lie group.

Example 4.2. Let $G$ be a compact group and let $K$ be a nonnormal subgroup so that $T_{\alpha} \mid K$ contains $K \rightarrow\{1\}$ at most once for each $\alpha \in \hat{G}$; equivalently, $\int_{K} \chi_{\alpha} d m_{K}=1$ or 0 , all $\alpha \in \hat{G}$. Then the space $H$ of two-sided cosets of $K$ is a P*-hypergroup. Indeed the space $C_{K K}(G)=\left\{f \in C(G): f\left(k x k^{\prime}\right)=f(x)(x \in G\right.$, $\left.\left.k, k^{\prime} \in K\right)\right\}$ is spanned by the spherical functions $\phi_{\alpha}=\chi_{\alpha} * m_{K}$ (see [5, p. 103]). Further $C_{K K}(G)$ is an algebra under the pointwise operations and its maximal ideal space is $H$. Here $M(H)$ is identified with $M_{K K}(G)=\left\{\mu \in M(G): m_{K} * \mu\right.$ * $m_{K}=\mu$, a commutative subalgebra of $M(G)$. As in Example 4.1, $H$ is a *hypergroup. Further $\phi_{\alpha} \phi_{\beta}$ is a linear combination of $\left\{\phi_{\gamma}: m_{\alpha \beta}(\gamma)>0\right\}$, and since $\phi_{\alpha} \phi_{\beta}$ is positive definite, it is a convex (finite) combination. Hence $H$ is a $\mathrm{P}^{*}$ hypergroup.

One example of this situation is $G=S O(n), K=S O(n-1)$ (where $S O(n)$ is the rotation group on $\mathbf{R}^{n}$ ). The spherical functions are ultraspherical polynomials of half-integer indices. The measure algebra has been studied in [4] or see [5, p. 107]. Another example is $G=U(n), K=U(n-1)$ (where $U(n)$ is the unitary group on $\mathbf{C}^{n}$ ). The spherical functions involve Jacobi polynomials.

Example 4.3. Let $\nu>0$ and let $P_{n}^{\nu}$ be the ultraspherical polynomial of degree $n$ and index $\nu$, normalized to have $P_{n}^{\nu}(1)=1$. Gegenbauer showed that for each pair $x, y \in[-1,1]$ there exists a positive measure $\lambda(x, y)$ on $[-1,1]$ such that $P_{n}^{\nu}(x) P_{n}^{\nu}(y)=\int_{-1}^{1} P_{n}^{\nu} d \lambda(x, y)$. With these measures [-1, 1] becomes a hypergroup with $\left\{P_{n}^{\nu}\right\}$ as the characters. The invariant measure is $a_{\nu}\left(1-t^{2}\right)^{\nu-1 / 2} d t$ (where $\left.a_{\nu}=\left(\int_{-1}^{1}\left(1-t^{2}\right)^{\nu-1 / 2} d t\right)^{-1}\right)$. Further $P_{m}^{\nu} P_{n}^{\nu}=\sum_{k=|m-n|}^{m+n} c(m, n ; k) P_{k}^{\nu}$ with $c(m, n ; k)$ $\geq 0$. Thus we have a $P^{*}$-hypergroup. For further details see Hirschman [9] or Askey and Hirschman [17].

Example 4.4. Suppose $H$ is a linearly independent set of continuous functions on a compact Hausdorff space with the following properties:

(1) the span of $H$ is dense in $C(X)$;

(2) $1 \in H$;

(3) there exists a point $p_{0} \in X$ such that $\|f\|_{\infty}=f\left(p_{0}\right)=1$ for all $f \in H$;

(4) $f g=\sum_{h \in H} n(f, g ; h) h(f, g \in H)$, where finitely many $n(f, g ; h)>0$ and the rest are zero. 
Conditions (3) and (4) together imply that $f g \in$ co $H(f, g \in H)$. Then $H$ has the structure of a discrete hypergroup with an identity (the function 1). Further $X$ is embedded in $\hat{H}$ by associating the evaluation map to each $x \in X$.

Example 4.5. Suppose that in addition to the properties of Example 4.4 the following hold:

(5) there exists $m \in M_{p}(X)$ so that $H$ is an orthogonal basis for $L^{2}(m)$;

(6) $f \in H$ implies $\bar{f} \in H$;

then $H$ is a discrete ${ }^{*}$-hypergroup. The proof of this is the same as used in $\S 3$ for $\hat{H}$.

Examples of such situations are given by Jacobi polynomials $\left\{P_{n}^{(\alpha, \beta)}: n\right.$ $=0,1,2, \ldots\}$ for $\alpha, \beta>-1, \alpha+\beta \geq-1$, by a recent result of Gasper [6].

Askey has also considered algebras of Jacobi polynomials. See his survey paper [1] for further references. Igari and Uno [10] have found the maximal ideal space (as hoped for, $[-1,1]$ ) of the algebra of absolutely convergent Jacobi series.

Example 4.6. Let $\left\{a_{i j}\right\}_{i, j=0}^{m}$ be a nonsingular $(m+1) \times(m+1)$ complex matrix with the following properties:

(1) $a_{0 j}=a_{i 0}=1$, for all $i, j$;

(2) $\left|a_{i j}\right| \leq 1$, all $i, j$;

(3) for $1 \leq i, j \leq m$, there exist nonnegative real numbers $n(i, j ; k)(0 \leq k$ $\leq m)$ such that $a_{i s} a_{j s}=\sum_{k=0}^{m} n(i, j ; k) a_{k s}(0 \leq s \leq m)$.

Consider the rows as being a set $H$ of functions on a space with $(m+1)$ points, then $H$ satisfies the hypotheses of Example 4.4. Further the characters are given by the columns of $\left\{a_{i j}\right\}$ (by a dimension argument, $\hat{H}$ can have no more than $m+1$ points).

We give an example of such a matrix for which $\hat{H}$ is not a hypergroup:

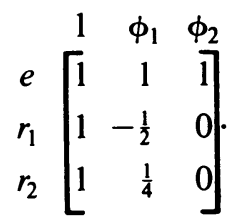

We see that $n(1,1)=(0,0,1), n(1,2)=\left(0, \frac{1}{2}, \frac{1}{2}\right)$, and $n(2,2)=\left(0, \frac{1}{4}, \frac{3}{4}\right)$. However $\phi_{1} \phi_{1}=\frac{1}{8}\left(\phi_{0}-2 \phi_{1}+9 \phi_{2}\right)$. By way of illustration of Theorem 1.12, the minimal ideal is $\left\{r_{1}, r_{2}\right\}$, and the invariant measure is $\left(0, \frac{1}{3}, \frac{2}{3}\right)$. Observe that $H$ is not a ${ }^{*}$ hypergroup.

Example 4.7. Let $H$ be given by the matrix

$$
\left[\begin{array}{rrr}
1 & 1 & 1 \\
1 & -1 & 0 \\
1 & 1 & \alpha
\end{array}\right],
$$

where $-1 \leq \alpha<0$. It can be shown that $H$ satisfies the hypotheses of 4.5 , and is in addition a $\mathrm{P}^{*}$-hypergroup, with invariant measure $(-\alpha / 2(1-\alpha), 1 / 2$, 
$1 / 2(1-\alpha)$ ). For $\alpha=-1 / 2$ this is essentially the character table of $S_{3}$, the symmetric group on 3 letters.

Vere-Jones has studied similar situations in [16].

Example 4.8. Let $H$ be given by the matrix

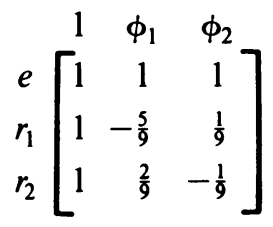

It can be shown that $H$ satisfies the hypotheses of 4.5 ; indeed

$$
\begin{aligned}
r_{1}^{2} & =(1 / 9) e+(8 / 9) r_{2}, \\
r_{1} r_{2} & =(4 / 9) r_{1}+(5 / 9) r_{2}, \\
r_{2}^{2} & =(1 / 18) e+(5 / 18) r_{1}+(2 / 3) r_{2} .
\end{aligned}
$$

Further $H$ is a ${ }^{*}$-hypergroup, since the characters are orthogonal with respect to the invariant measure $(1 / 28,9 / 28,9 / 14)$ (see the proof of Theorem 3.17). However $\phi_{1}^{2}=(1 / 6) 1-(2 / 27) \phi_{1}+(49 / 54) \phi_{2}$. Thus there exists a finite (compact) *-hypergroup which is not $\mathrm{P}^{*}$.

The author thanks his colleague Leonard Scott for providing this example.

\section{REFERENCES}

1. R. Askey, Orthogonal polynomials and positivity, Studies in Appl. Math., no. 6, SIAM, Philadelphia, Pa., 1970, pp. 64-85.

2. A. P. Dičman (Dietzmann), On the multigroup of complete conjugate sets of elements of a group, $C$. R. (Dokl.) Acad. Sci. URSS 49 (1946), 315-317. MR 7, 511.

3. N. Dunford and J. T. Schwartz, Linear operators. I: General theory, Pure and Appl. Math., vol. 7, Interscience, New York, 1958. MR 22 \#8302.

4. C. Dunkl, Operators and harmonic analysis on the sphere, Trans. Amer. Math. Soc. 125 (1966), 250-263. MR 24 \#3224.

5. C. Dunkl and D. Ramirez, Topics in harmonic analysis, Appleton-Century-Crofts, New York, 1971.

6. G. Gasper, Linearization of the product of Jacobi polynomials. I, Canad. J. Math. 22 (1970), $171-175$.

7. I. Glicksberg, Convolution semigroups of measures, Pacific J. Math. 9 (1959), 51-67. MR 21 \#7405.

8. A. Grothendieck, Critères de compacité dans les espaces fonctionnels généraux, Amer. J. Math. 74 (1952), 168-186. MR 13, 857.

9. I. Hirschman, Harmonic analysis and ultraspherical polynomials, Proc. Conference on Harmonic Analysis, Cornell University, Ithaca, N.Y., 1956.

10. S. Igari and Y. Uno, Banach algebra related to the Jacobi polynomials, Tôhoku Math. J. 21 (1969), 668-673.

11. B. Levitan, The application of generalized displacement operators to linear differential equations of the second order, Uspehi Mat. Nauk 4 (1949), no. 1 (29), 3-112; English transl., Amer. Math. Soc. Transl. (1) 10 (1962), 408-541. MR 11, 116. 
12. M. A. Naĭmark, Normed rings, GITTL, Moscow, 1956; rev. ed., English transl., Noordhoff, Groningen, 1964. MR 19, 870; MR 34 \# 4928.

13. D. Ragozin, Central measures on compact simple Lie groups, J. Functional Analysis 10 (1972), 212-229.

14. D. Rider, Central idempotent measures on unitary groups, Canad. J. Math. 22 (1970), 719-725. MR 41 \#8929.

15. M. Rosenblatt, Limits of convolution sequences of measures on a compact topological semigroup, J. Math. Mech. 9 (1960), 293-305; Addendum, ibid. 10 (1961), 681. MR 22 \#9544; MR 22 \#2547.

16. D. Vere-Jones, Finite bivariate distributions and semigroups of nonnegative matrices, Quart. J. Math. Oxford Ser. (2) 22 (1971), 247-270.

17. R. Askey and I. Hirschman, Jr. Weighted quadratic norms and ultraspherical polynomials. I, Trans. Amer. Math. Soc. 91 (1959), 294-313. MR 21 \#6494.

Department of Mathematics, University of Virginia, Charlottesville, Virginia 22904 